\title{
Force-Velocity Relations in the Intact Dog Heart*
}

\author{
Herbert J. Levine and Naphtali A. Britman $\dagger$ \\ (From the Pratt Clinic-New England Center Hospital and the Department of Medicine, \\ Tufts University School of Medicine; Boston, Mass.)
}

Few subjects in medicine have attracted as much attention from physiologists and clinical investigators alike as has the study of the mechanics of cardiac contraction. Our current understanding of this subject is the result of studies of both isolated muscle and the intact heart. The geometric simplicity of parallel fiber muscle strips and the ability to measure and control specific parameters in isolated muscle preparations have provided the physiologist with a means of systematic analysis of the behavior of the contractile element. Working with isotonic contractions of skeletal muscle strips, Hill described the most fundamental mechanical property of the contractile element: namely, the hyperbolic relation between force generated and the velocity of shortening $(1,2)$. In his analysis of muscle mechanics, Hill visualized force generation as the stretching of an elastic component that was functionally in series with the contractile element. More recently, Abbott and Mommaerts (3) and Sonnenblick (4) have demonstrated the force-velocity relationship in the cat papillary muscle and have pointed up basic differences between cardiac and skeletal muscle in the behavior of this relationship during specific interventions.

Studies of the mechanics of contraction in the intact heart have utilized other measured parameters. The Frank-Starling mechanism $(5,6)$ has provided us with the most useful index of the functional state of the intact heart. In the construction of a ventricular function curve, however, the contractile effort of the heart is represented solely by external stroke work, and the total work of the contractile element may not be

* Submitted for publication July 9, 1963; accepted February 27, 1964.

This work was supported by U. S. Public Health Service research grant H-7139 from the National Heart Institute.

† Postdoctoral fellow of the National Heart Institute, U. S. Public Health Service. fully realized (7). Although the time derivatives of stroke work, isometric pressure, and stroke volume further help to characterize the contractile effort of the beating heart, an examination of the fundamental relationship between force and velocity of the contractile element throughout the course of a single systole has not been reported. In a recent discussion of muscle mechanics, however, Fry has presented preliminary data on the instantaneous relation between muscle tension, shortening velocity, and fiber length in the intact heart (8).

The purpose of this paper is to describe a method for examining the instantaneous relationship between contractile element velocity and force in the beating heart and to show that the inverse force-velocity relationship observed in isolated muscle is operative in the intact heart. Analysis of this relationship in the intact animal provides a means of interpreting cardiac function in terms of basic muscle mechanics.

\section{Methods}

Methods and experimental design. Sixty-three studies were carried out in 29 mongrel dogs, weighing from 14 to $24 \mathrm{~kg}$, anesthetized with sodium pentobarbital, 25 $\mathrm{mg}$ per $\mathrm{kg}$, and chloralose, 50 to $70 \mathrm{mg}$ per $\mathrm{kg}$, with or without premedication with morphine sulfate, $3 \mathrm{mg}$ per kg. Respirations were driven by a Harvard pump attached to a cuffed endotracheal tube. Left ventricular and aortic arch pressures were recorded via no. 7 Goodale-Lubin catheters connected directly to Statham P23Db strain gauges and recorded on an Electronics for Medicine photographic recorder at paper speeds of $100 \mathrm{~mm}$ per second. The resonant frequency of this catheter system was at $54 \mathrm{cps}$, and the distortion at $15 \mathrm{cps}$ was $+5 \%$. On some occasions frequencies of greater than 25 cycles per second were filtered. Intrathoracic pressure was recorded via a polyethylene catheter passed percutaneously into the chest, and in some experiments this pressure was subtracted electrically from the left ventricular diastolic pressure. A catheter was placed in the pulmonary artery and cardiac output determined by the direct Fick method. Oxygen determinations were 

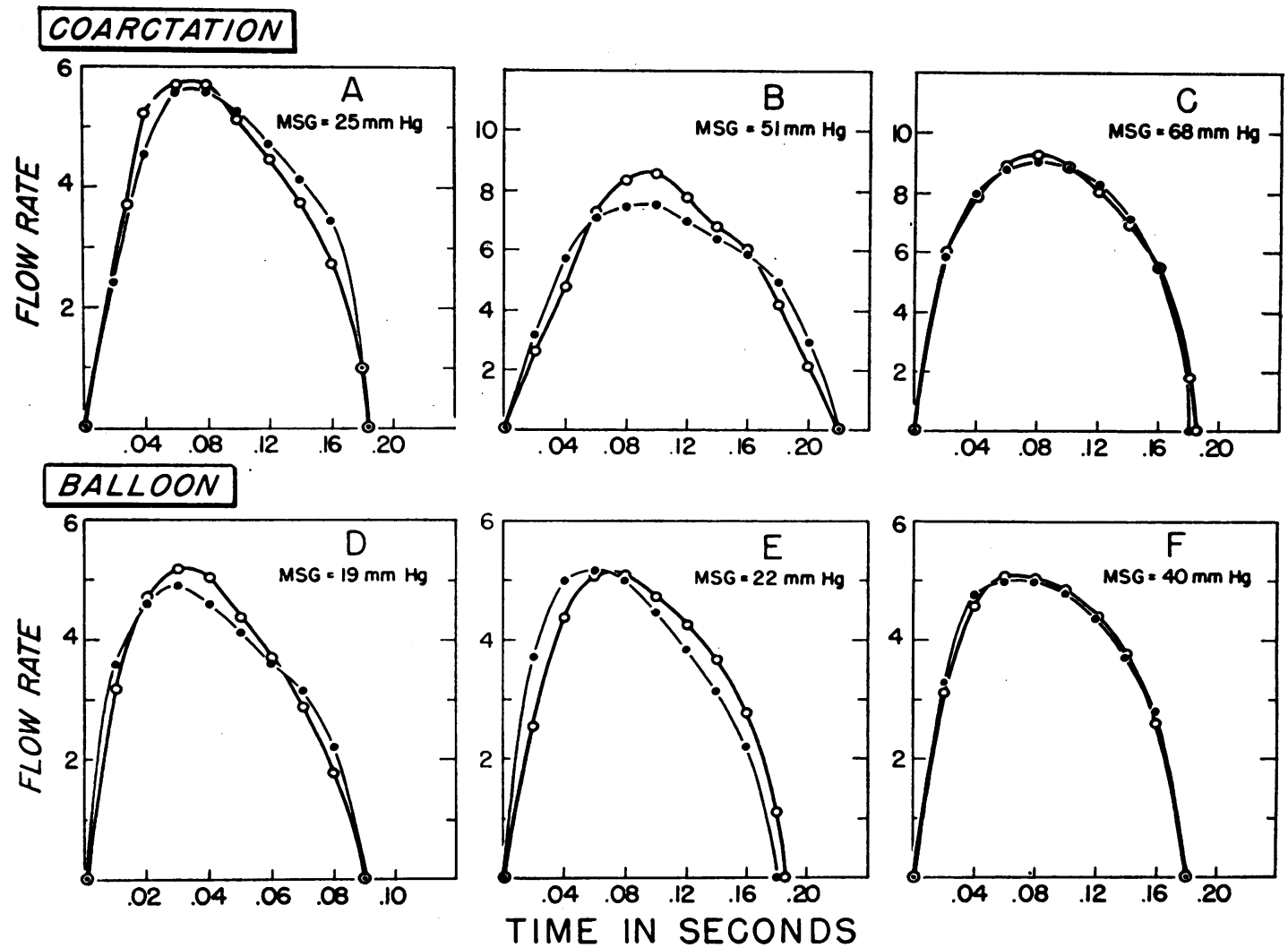

Fig. 1. Comparison of the time COURSE OF AORTIC Flow RATE DETERMINEd bY: 1) THE SQUARE ROOT OF THE INSTANTANEOUS PRESSURE GRADIENT (SOLID CIRCLES) AND 2) A SQUARE WAVE ELECTROMAGNETIC FLOWMETER (OPEN CIRCLES). MSG = mean systolic gradient. Flow rate is given in arbitrary units. In $\mathrm{A}, \mathrm{B}$, and $\mathrm{C}$ the gradient was produced by surgical coarctation, and in D, E, and F, by an intra-aortic balloon. See text.

performed by the manometric method of Van Slyke and Neill (9).

A thermistor ${ }^{1}$ was introduced into the arch of the aorta by a femoral artery catheter and left ventricular volume determined by the thermal dilution technic $(10$, 11). Two to four $\mathrm{ml}$ of room temperature saline was injected into the left ventricle and the "step function" of aortic temperature recorded. One to three thermal dilution curves were recorded during each experimental state, and depending upon the degree of ventricular emptying, 2 to 8 individual end-systolic volume/end-diastolic volume (ESV/EDV) ratios were calculated from each curve. The average $\mathrm{ESV} / \mathrm{EDV}$ ratio for the entire study was $79 \%$ (40 to $95 \%$ ). Mean ESV was 51.6 $\mathrm{ml}(12$ to $136 \mathrm{ml}$ ), and mean EDV was $62.9 \mathrm{ml}$ (17 to $146 \mathrm{ml}$ ). The variations in $\mathrm{ESV} / \mathrm{EDV}$ from a beat to beat analysis of each curve have been expressed as a percentage of difference from the average of each experimental period, regardless of sign. Thus, the average of absolute deviation in the ESV/EDV ratio was $2.1 \%$, which agrees closely with that reported in the literature

1 Victory Engineering Corp., Union, N. J.
(11). The standard error of the EDV measurement was calculated for each curve and expressed as SE/EDV. This error varied from $0.2 \%$ to $24 \%$ with an average of $5.7 \%$.

To derive the instantaneous flow rate during a single systole, an arterial gradient was produced in the proximal ascending aorta by two different technics. In 9 animals, surgical coarctation of the aorta was produced via a sternal-splitting thoracotomy by introducing an umbilical tape tourniquet around the aorta 1.5 to $2.0 \mathrm{~cm}$ above the coronary ostia. This tape was led out of the chest through a glass tube and could be tightened or loosened by spiraling of the tape through the tube with a perpendicular glass rod after the chest was closed. In the remaining animals, a single-barreled metal cannula with a distal balloon was introduced through a carotid artery and positioned just above the aortic valve. Because the latter technic obviated the surgical trauma of thoracotomy and dissection, it was used in the majority of experiments here reported. Generally, left ventricular end-diastolic and end-systolic volumes were measured before aortic obstruction. 
When a given degree of aortic resistance was produced, sufficient time was allowed for a steady state to be reached as evidenced by a constant arterial gradient and left ventricular end-diastolic pressure (LVed). Cardiac output, pressure, heart rate, and left ventricular volume were then measured simultaneously. Changes in left ventricular pressure or output or both were achieved by varying the degree of aortic obstruction. Volume loading was produced by the rapid transfusion of 500 to $1,500 \mathrm{ml}$ of whole blood, sufficient to produce elevation of the left ventricular end-diastolic pressure. As an inotropic intervention, isoproterenol was infused as a constant rate ( 1 to $5 \mu \mathrm{g}$ per minute). No attempt was made in these experiments to control the reflexes or hormonal milieu of the animal. Thus, the response of the heart to a specific intervention represents that of an intact, anesthetized dog.

At the conclusion of each study, the left ventricle with the interventricular septum was dissected from the remaining chambers, valves, and chordae, and was weighed.

Calculations and definitions. Stroke volume was derived by dividing the cardiac output by the heart rate, and end-diastolic and end-systolic volumes were determined from the thermal dilution curve and the stroke volume $(10,11)$. The instantaneous pressure gradient produced in the manner described above was used to calculate the instantaneous flow rate during the course of a single systole utilizing Torricelli's orifice equation for high velocity, turbulent flow $(12,13)$. . Thus, the instantaneous flow rate is proportional to the square root of the instantaneous pressure gradient. The square root of the instantaneous pressure gradient, at each $0.02 \mathrm{sec}-$ ond of systole, was plotted against time and the area under this curve equated to stroke volume. In this manner the constant relating flow rate to the square root of the pressure gradient was obtained. That portion of the stroke volume ejected up to each interval was derived by direct planimetry of the area under the corresponding portion of the curve. The instantaneous volume was derived by subtracting the volume ejected up to each point in systole from the end-diastolic volume. Assuming the ventricle to be spherical, the instantaneous radius was calculated from the equation $V=4 \pi r^{3} / 3$. The validity of this technic as a measurement of instantaneous flow rate was tested in the following manner. The probe of a square wave electromagnetic flowmeter ${ }^{2}$ was placed on the proximal aorta, just distal to the site of surgical coarctation (or balloon obstruction). The time course of aortic root flow, as measured by both the flowmeter and the square root of the pressure gradient, was plotted on graph paper. As the area under both these curves represented stroke volume, the planimetered area of one curve was forced to equal that of the other, and the form of the two curves compared. Over a wide range of pressure gradients (15 to $70 \mathrm{~mm}$ $\mathrm{Hg}$ ), produced by either surgical coarctation or balloon

2 Model 300, Carolina Medical Electronics, WinstonSalem, N. C.

\section{3-COMPONENT MODEL}

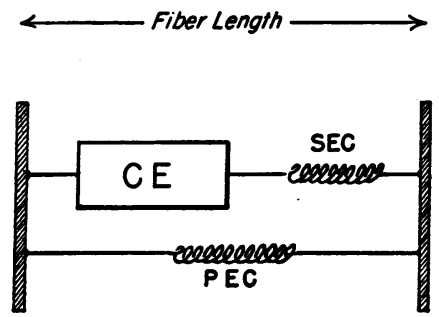

Fig. 2. Three-component Model AFter Hill (2). Contractile element, CE; series elastic component, SEC; parallel elastic component, PEC. See text.

obstruction, the two curves were found to be virtually superimposable (Figure 1). Occasionally, aortic flow as measured by the flowmeter was demonstrated immediately after aortic valve closure and was due to recoil of the aortic reservoir proximal to the flowmeter. In such instances (E, Figure 1) the flowmeter curve was slightly delayed when compared to that derived from the square root of the pressure gradient.

The circumferential fiber shortening rate (CFSR), defined as the rate of fiber shortening at the equator of a sphere, was calculated at each 0.02 -second interval during systole, as flow rate $/ 2 \mathrm{r}^{2} 3$ and is expressed in centimeters per second. Myocardial tensile force $(F)$ was calculated at similar intervals at $F=\pi r^{2} P$, where $F=$ total tangential myocardial force in dynes, $P=$ intraventricular pressure in dynes per $\mathrm{cm}^{2}$, and $r=$ internal radius of the left ventricle in centimeters ( 7$)$. The force-time curve during systole was plotted, and the slope $(\mathrm{dF} / \mathrm{dt})$, expressed as dynes per second, was measured directly at each time interval.

To compare $F$ and CFSR in left ventricles of different sizes, the proportions of the unstretched ventricle were assumed to be similar from dog to dog. Thus, the cross-sectional area of the muscle wall would vary as the two-thirds power of left ventricular weight, and the circumference (fiber length) would vary as the onethird power of this weight. Since force generation in muscle is dependent upon the number of contractile units that exist in parallel (cross-sectional area) and not the number that exist in series, myocardial tensile force from dog to dog was expressed as F per gram?. CFSR, on the other hand, is dependent upon the number of contractile units that exist in series (fiber length), and therefore CFSR from dog to dog was expressed as CFSR per gram?

In our analysis of the muscle mechanics of the intact left ventricle, we have utilized the three-component model of Hill (2). As shown in Figure 2, the muscle fiber is

3 By differentiating the volume equation for a sphere, $d V / d t$ (flow rate) $=4 \pi r^{2} d r / d t$. Since circumferential fiber length is equal to $2 \pi \mathrm{r}, \mathrm{CFSR}=2 \pi d r / d t$. By substitution, $\mathrm{CFSR}=(d V / d t) / 2 r^{2}$. 

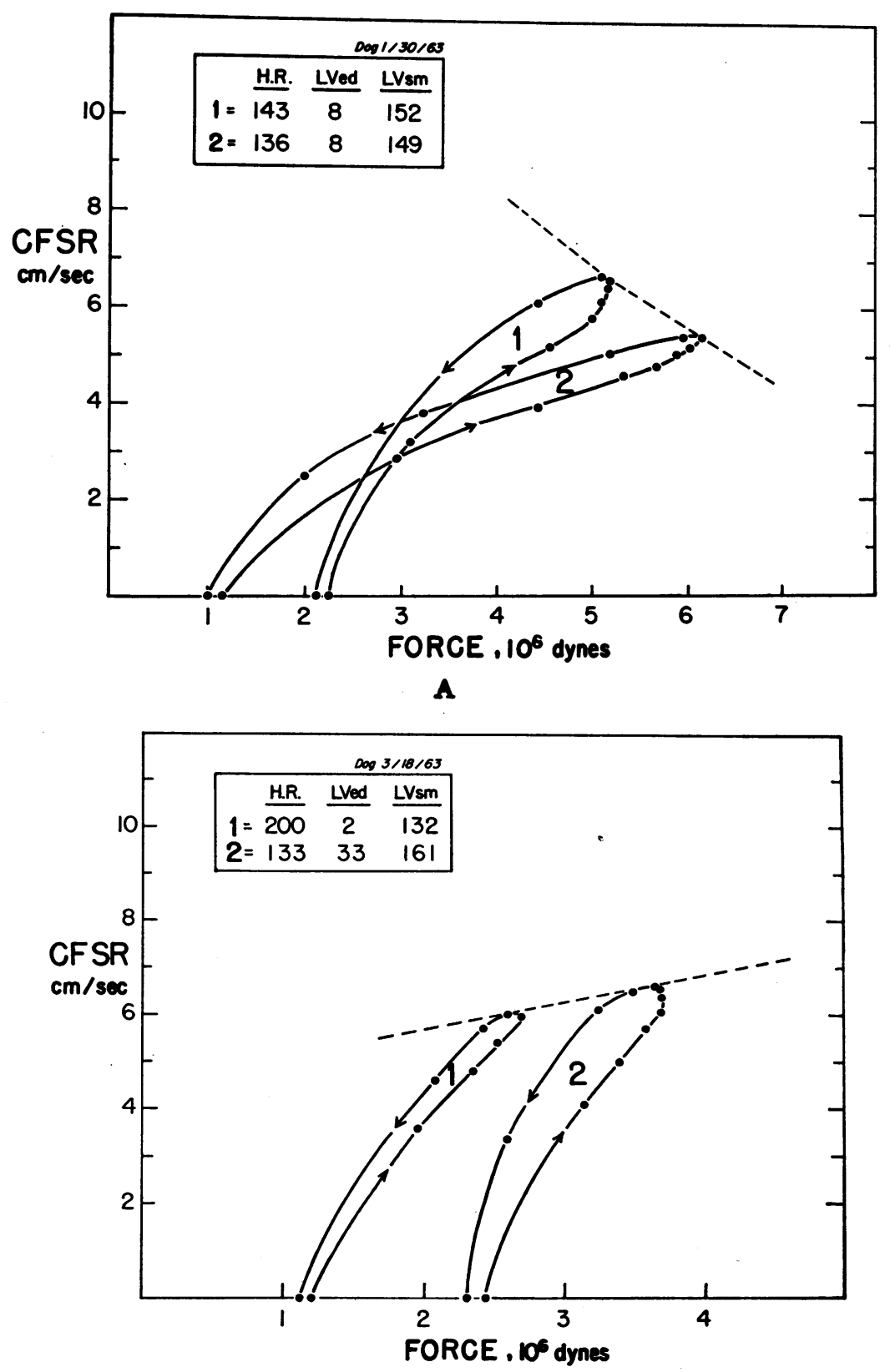

B

Fig. 3. (Continued on opposite page.)

considered functionally to be composed of two components in series and a third in parallel with these. The former consists of an active contractile element (CE) and a passive series elastic component (SEC); the latter is represented by a passive, parallel elastic component (PEC). The PEC does not play a role in the mechanics of contraction, but as the relaxed $\mathrm{CE}$ is considered to be freely extensible, the $\mathrm{PEC}$ is mainly responsible for the tension of the stretched, resting muscle. It is apparent from this model that the contractile effort of the fiber resides wholly within the $\mathrm{CE}$ and that this may be manifest as isotonic fiber shortening (with a constant length of the SEC), as isometric tension development due to stretching of the SEC (with fiber length remaining constant), or as a combination of both fiber shortening and tension development.

During the ejection phase of a normal cardiac systole, the contractile element shortening velocity (Vce) must 
Fig. 3. FORCE-CIRCUMFERENTIAL FIBER SHORTENING RATE (CFSR) RELATIONS DURING THE SYSTOLIC EJECTION PERIOD. A. A minor change in the degree of aortic obstruction was produced. Left ventricular enddiastolic (LVed) and systolic (LVsm) pressures remained unchanged, and heart rate (HR) changed very little. Although the two points at peak force describe an inverse relation between force and velocity, it is not possible to say that they lie on the same or a closely related force-velocity curve. B. Volume loading with whole blood produced a marked increase in LVed pressure, cardiac slowing, a rise in LVsm pressure, and a lengthened duration of systole. Although the two points of peak force cannot lie on the same inverse forcevelocity curve, whether one or both intercepts have been changed cannot be determined by this analysis. C. An inotropic intervention (isoproterenol) was associated in this study with a mild rise in LVed, although end-diastolic volume fell $19 \%$. Heart rate did not change significantly. Although the F-CFSR relationship at peak force was altered greatly, it is not possible to infer from these measurements that the force-velocity curve has been altered by isoproterenol (see Figure 10).

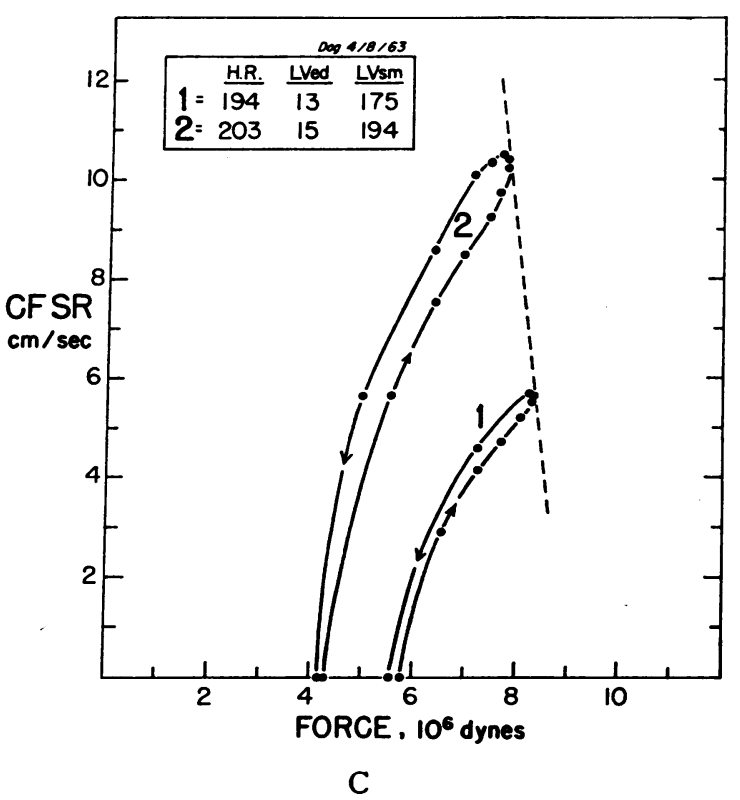

dynes $)] /\left[l_{0}\right.$ (centimeters) $]+\left[0.241\left(10^{6}\right.\right.$ dynes per centimeter) $\left.A_{0}\left(\mathrm{~cm}^{2}\right)\right] /\left[l_{0}\right.$ (centimeters) $]$, where $l_{o}=$ shortest end-diastolic circumferential fiber length and $A_{0}=$ cross-sectional area of muscle. The cross-sectional area of muscle was calculated by the addition of the measured left ventricular weight to the smallest end-diastolic volume recorded during the study. In this manner the radii of the concentric spheres representing the inner and outer limits of the ventricular wall were derived, and their difference represented mean wall thickness $(t)$. Crosssectional area of muscle was calculated at $22 \pi r_{0} t$, where $r_{o}=$ radius of the inner surface of the ventricle. $l_{o}$ was equal to $2 \pi r_{0}$. The smallest end-diastolic volume was used to approximate the resting dimensions.

Using the measured $\mathrm{dF} / \mathrm{dt}$ and the above approximation of $\mathrm{dF} / \mathrm{dl}$, a value of $\mathrm{dl} / \mathrm{dt}$ and thereby $\mathrm{Vce}$ was derived for each value of $F$ examined during the course of systole. In this fashion a force-velocity curve was constructed. Because of the obvious inaccuracies inherent in this derivation, a second technic for determining $\mathrm{dF} / \mathrm{dl}$ was employed. As mentioned above, the stiffness of the SEC has been shown to be constant in a given muscle for a given force. Thus, if the heart could be made to function on two different portions of the same force-velocity curve, the sum of CFSR and $\mathrm{dl} / \mathrm{dt}$ at each value of $F$ would be the same, although their ratio would be different. To achieve this end, a small change in the aortic obstruction was produced, in such a manner that changes in end-diastolic volume and heart rate were not observed. Since the Vce is equal to the sum of the CFSR and the $\mathrm{dl} / \mathrm{dt}$, the value of $\mathrm{dF} / \mathrm{dl}$ at each point of force development common to both states was derived as follows: $\mathrm{CFSR}_{1}+(d l / d F)(d F / d t)_{1}=\mathrm{CFSR}_{2}+(d l /$ $d F)(d F / d t)_{2}$.

4 Taken from Figure 17 (14). 

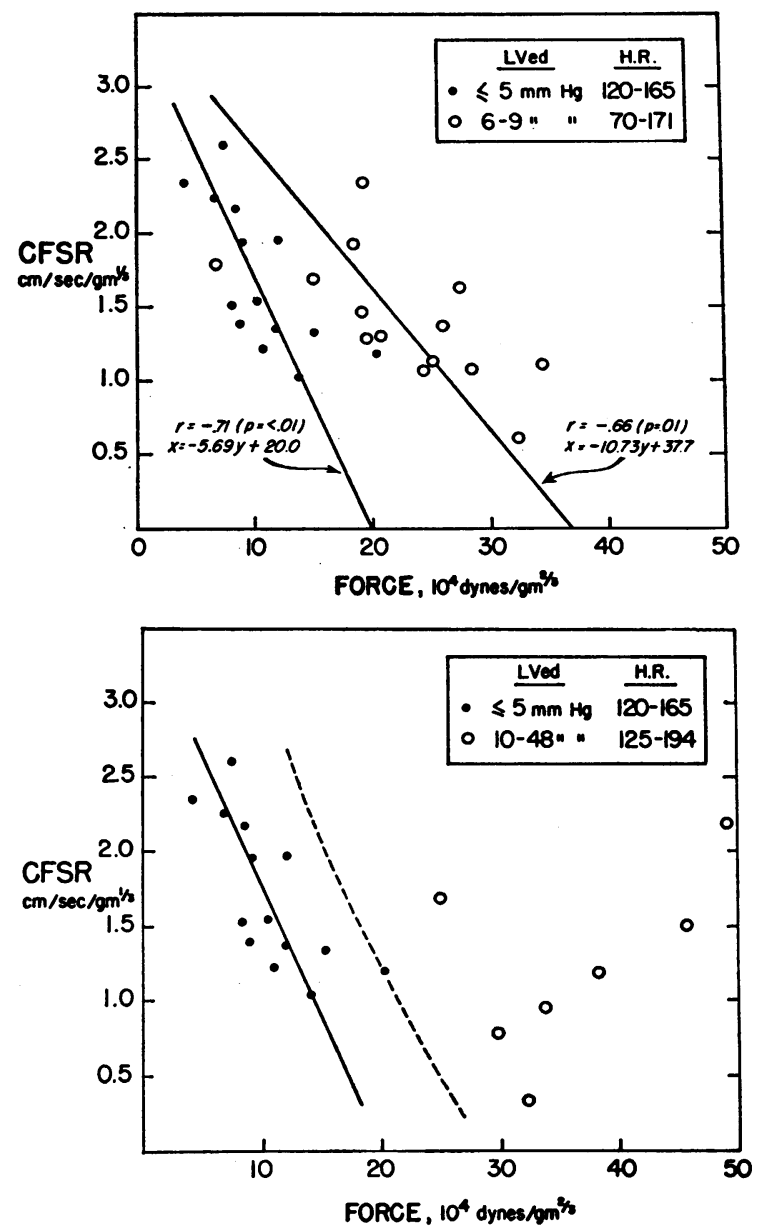

Fig. 4. INSTANTANEOUS FORCE-CIRCUMFERENTIAL FIBER SHORTENING RATE RELATIONS AT PEAK FORCE, CORRECTED FOR DIFFERENT LEFT VENTRICULAR WEIGHTS. The solid dots represent all studies where LVed $\leqslant 5 \mathrm{~mm} \mathrm{Hg}$ and heart rate was between 120 and 165 beats per minute. Upper. The open circles represent those studies where a mild elevation of LVed was observed (6 to $9 \mathrm{~mm} \mathrm{Hg}$ ). Lower. The open circles represent studies in which marked elevations in LVed (10 to $48 \mathrm{~mm} \mathrm{Hg}$ ) were produced by volume loading. The interrupted line represents the control group $95 \%$ confidence band for a new observation. See text.

In this manner a single force-velocity curve was constructed from the two points where $d F / d t=0^{5}$ and from all those points of force development from which a common value of $\mathrm{dF} / \mathrm{dl}$ could be derived.

${ }^{5} \mathrm{As} \mathrm{dF} / \mathrm{dt}$ is equal to zero at peak tension, the CFSR is equal to Vce only at this point during systole, and thus, when the anisotonic, anisometric contraction of the intact heart is examined in this fashion, the F-CFSR relationship at peak tension represents a true point on the force-velocity curve.
In the discussion of force-velocity curves, $F_{0}$ is defined as the extrapolated value of force at zero velocity and $V \max$ as the extrapolated velocity at zero load.

\section{Results}

Myocardial tensile force $(F)$ and circumferential fiber shortening rate (CFSR) relationships. In each experiment, the values for instantaneous $\mathrm{F}$ and CFSR were plotted at 0.02 -second intervals during the systolic ejection period. In 52 of 63 instances a counterclockwise loop with varying degrees of hysteresis was recorded. No hysteresis was found in 5 experiments, and a clockwise loop was noted in 6 . Upon the production of a specific intervention, a second loop was constructed, and the two points of peak tension were related to one another. Examples of this analysis are illustrated in Figure 3.

In Figure 3-A, a very minor intervention was induced. The aortic obstruction was increased very slightly, so that no change occurred in left ventricular end-diastolic pressure (LVed) or heart rate. The F-CFSR relationship at peak tension in these two loops suggests an inverse relationship between force and velocity. Even if this inverse relationship should apply to the beating heart, however, there is little reason to believe that these two points fall on the same or even a closely related inverse curve. In Figure 3-B volume loading sufficient to raise the LVed from

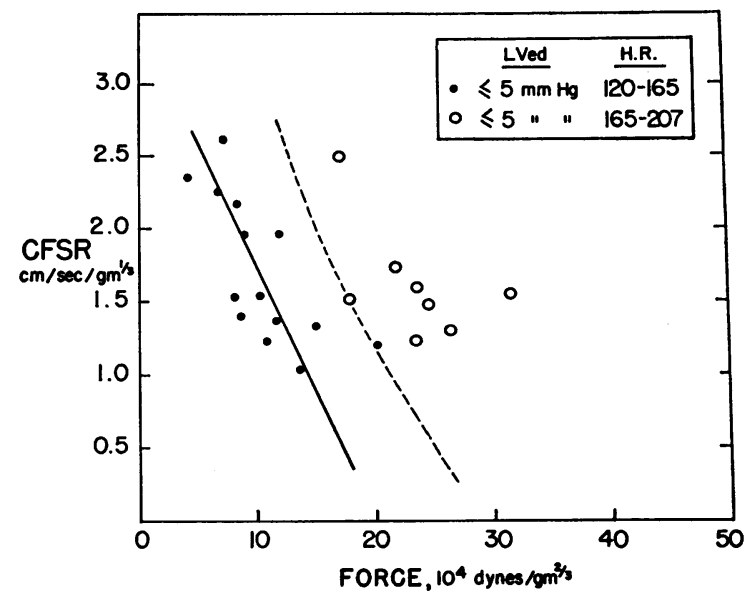

Fig. 5. See legend to Figure 4. The open circles represent instantaneous F-CFSR relations at peak force in those experiments in which heart rate was increased without an elevation in LVed. See text. 
2 to $33 \mathrm{~mm} \mathrm{Hg}$ has so changed the F-CFSR relationship at peak tension that these points clearly do not lie on the same inverse force-velocity curve. The effect of an inotropic intervention, isoproterenol, is presented in Figure 3-C. Although a major change in peak force and CFSR has been produced, it is not possible to say whether or how the force-velocity relationship during systole has been altered. Thus, examination of the F-CFSR relationship in paired observations is of little help in discerning even directional changes in the force-velocity relationship during systole. Since the F-CFSR loop of a single systole provides only one true point on the force-velocity curve, many such points from comparable studies were then analyzed.

Comparison of F-CFSR relations at peak tension. In Figures 4 and 5, the F-CFSR relations at peak tension are presented for each study, "normalized" for left ventricular weight (vide supra). When normalization was carried out according to left ventricular dimensions $\left(l_{o}\right.$ and $\left.2 \pi r_{0} t\right)$ rather than weight, little difference in the F-CFSR relations were observed. Because the measurement of left ventricular weight was simpler and involved less experimental error, it was adopted as the basis for comparing values of $F$ and CFSR in different dog hearts.

In 14 experiments the LVed pressure was between 0 and $5 \mathrm{~mm} \mathrm{Hg}$ and the heart rate between 120 and 165 beats per minute (mean $=145 \pm 11.7$ beats per minute) ${ }^{8}$ These 14 experiments constitute the control group (Figures 4 and 5). An inverse first order relationship between points of F-CFSR at peak force was found in this group with an $r$ of $-0.71(p<0.01)$. In a second group of 14 experiments (Figure 4-A), the LVed was mildly elevated ( 6 to $9 \mathrm{~mm} \mathrm{Hg}$ ); heart rate was not significantly different (mean $=136 \pm$ 33.8 beats per minute). In this group a first order relationship was found with an $r$ of -0.66 $(p=0.01)$. In both the control and the group with mild elevations in LVed, the $y$ intercepts of the regression lines were the same, whereas the $x$ intercepts were significantly different (by the $t$ test, $\mathrm{p}<0.01$ ). In 7 experiments (Figure 4-B) a major elevation in LVed was observed (10 to 48 $\mathrm{mm} \mathrm{Hg}$ ), but average heart rate was little changed

6 Values are mean $\pm \mathrm{SD}$.

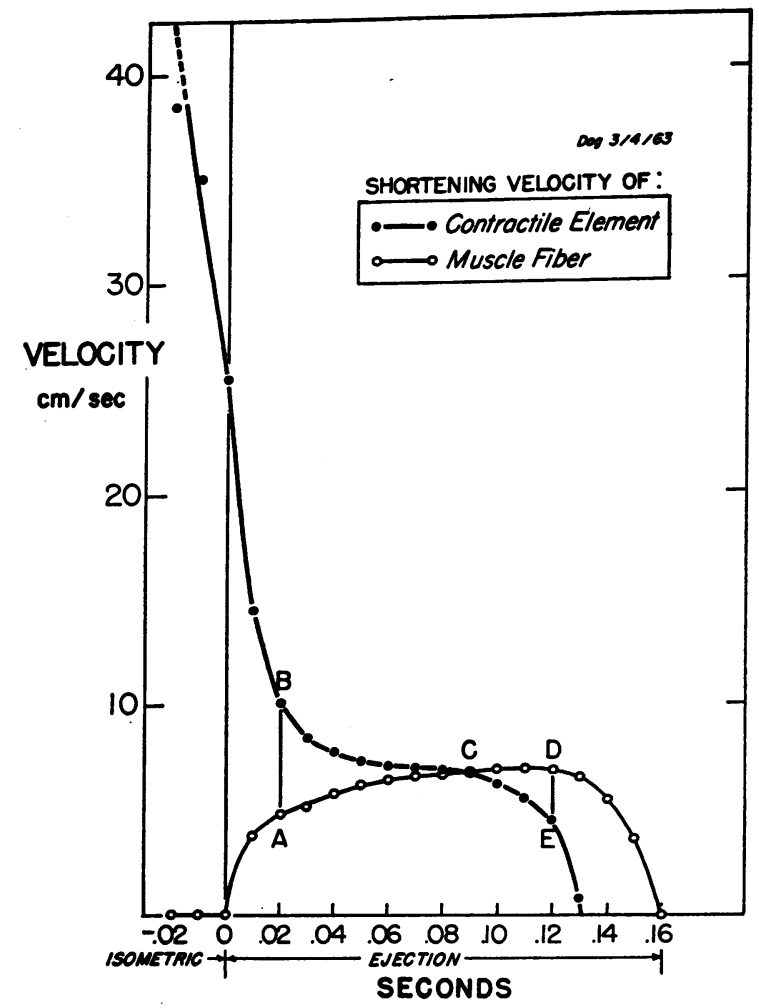

Fig. 6. Time course of CFSR and Contractile eleMENT SHORTENING VELOCITY (VCE) DURING SYSTOLE. Time zero is defined as the beginning of the ejection period. During the early isometric period a maximal Vce is rapidly achieved. Vee then falls and continues to do so as fiber shortening begins. At 0.02 seconds the fiber shortening velocity is equal to $A$, and the lengthening velocity of the SEC (dl/dt) is equal to $B$ minus $A$. At 0.09 seconds CFSR and Vce are the same (C), and $\mathrm{dl} / \mathrm{dt}=0$. Thereafter CFSR exceeds Vce as the SEC itself starts to shorten. Thus, at 0.12 seconds Vce is equal to $E$, and the shortening velocity of the SEC $(-\mathrm{dl} / \mathrm{dt})$ is equal to $\mathrm{D}$ minus $\mathrm{E}$. Beyond 0.13 seconds the shortening velocity of the SEC exceeds that of the fiber itself, and the contractile element begins to lengthen $(-\mathrm{Vce})$.

( $156 \pm 27.5$ beats per minute). All 7 points were found to lie outside of the $95 \%$ confidence band for a new observation (control group).

In Figure 5 the effect of an increased heart rate is examined. In 8 experiments the heart rate ranged from 165 to 207 (mean $=193 \pm 31.1$ beats per minute), and the LVed remained in the same range as the control group ( $\leqslant 5 \mathrm{~mm} \mathrm{Hg}$ ). At comparable levels of CFSR, a greater peak force was achieved in the higher heart rate group than in the control group (all values beyond the 


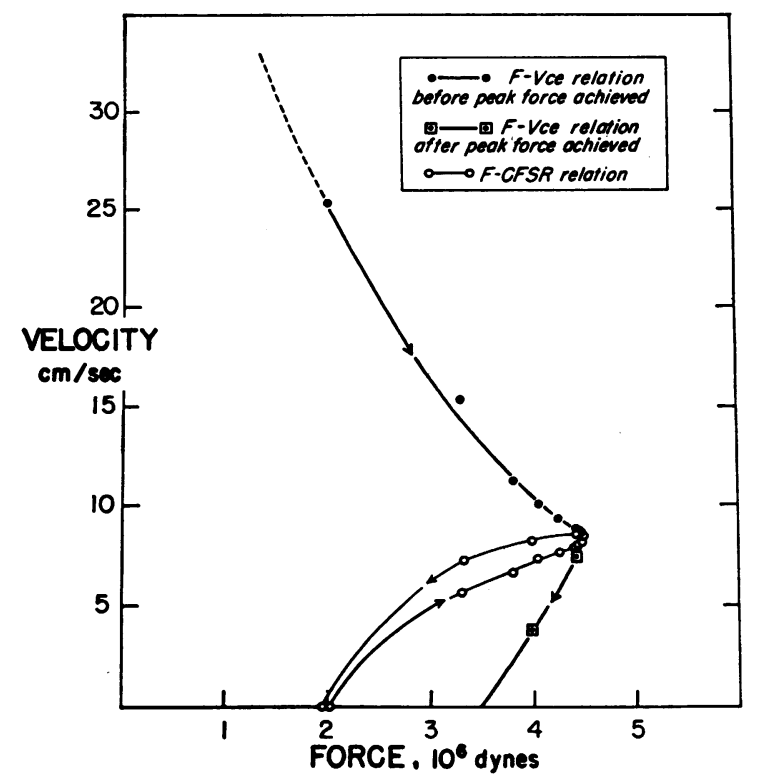

Fig. 7. ForCe-velocity AND FORCE-CFSR RELATIONS DURING SYSTOLE. During the first part of systole, until the point of peak force, an inverse relation between force and contractile elements velocity is observed. Thereafter as force decreases, contractile element velocity continues to fall and actually becomes negative before the end of the ejection period. The vertical distance between corresponding points of Vce and CFSR represents the velocity of the SEC.

control group 95\% confidence band for a new observation).

Relationship between CFSR and contractile element shortening velocity $(V c e)$. Figure 6 depicts the time course of CFSR and Vce during systole of a typical experiment. The values of Vce were obtained as the sum of the CFSR and the $\mathrm{dl} / \mathrm{dt}$ of the series elastic component, and the latter was derived from extrapolation of the normalized Sonnenblick equation for the cat papillary muscle to the specific dimensions of the dog's left ventricle. During the isometric period, Vce rapidly fell from an early maximum as wall force and the stiffness of the SEC rise. ${ }^{7}$ As ejection began and the fiber shortened, Vce continued to

7 The calculation of Vce during the isometric period is fraught with error due both to an inability to accurately measure the rate of pressure rise during the early isometric period and to the large error introduced by the parallel elastic component in the stiffness calculation at low tensions. It is included here solely to indicate gross directional changes in Vce during early systole. fall but less rapidly than did the lengthening rate of the SEC. At the point of maximal tension (C, Figure 6), Vce and CFSR were the same. Thereafter, CFSR exceeded the Vce, the difference being due to "recoil" of the SEC $(-\mathrm{dl} / \mathrm{dt})$. During this stage of systole, shortening of the SEC actually assisted the contractile element in shortening of the fiber. The peak CFSR occurred slightly later in systole than peak tension in most of these studies.

Force-velocity relationships. In most experiments the instantaneous values of $F$ and Vce were plotted for a single cardiac systole as shown in Figure 7. In addition the corresponding F-CFSR relationship is shown. In this manner, at any one time during systole, the fraction of contractile element velocity expressed as fiber shortening and that expressed as tension generation may be assessed separately. From the onset of fiber shortening until peak force was achieved,

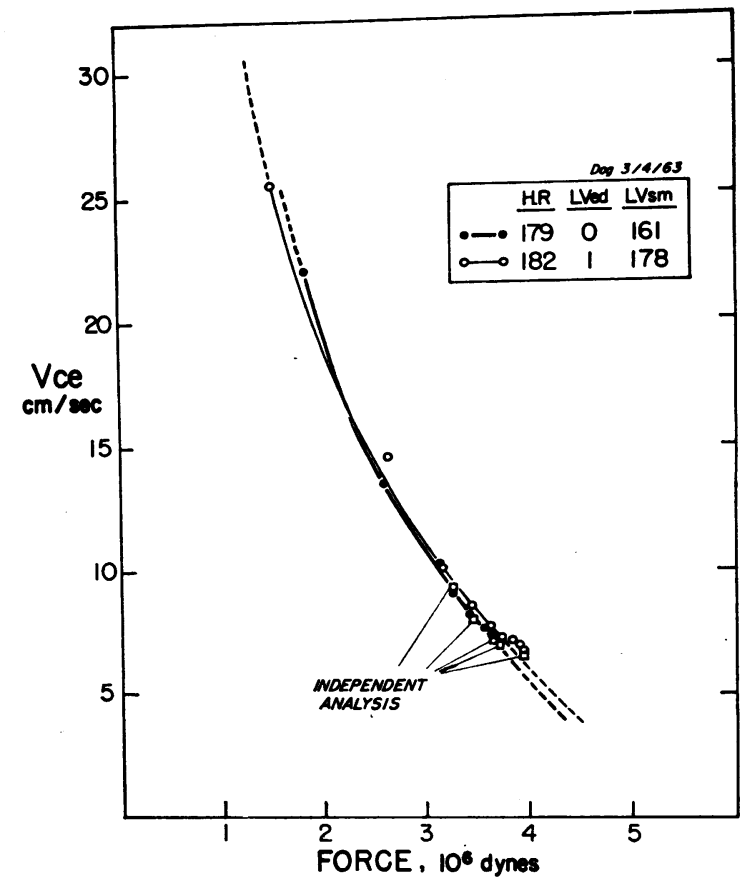

Fig. 8. INDEPENDENT ANALYSIS OF FORCE-VELOCITY CURVE. The force-velocity curves of two closely related hemodynamic states, derived utilizing the normalized "stiffness" equation, are shown to be virtually identical. The stiffness of the SEC $(\mathrm{dF} / \mathrm{dl})$ was calculated by an independent technic (see text) for each of 5 points of force, common to both states. Note that the forcevelocity curve constructed from these 5 points is indistinguishable from the other two curves. 


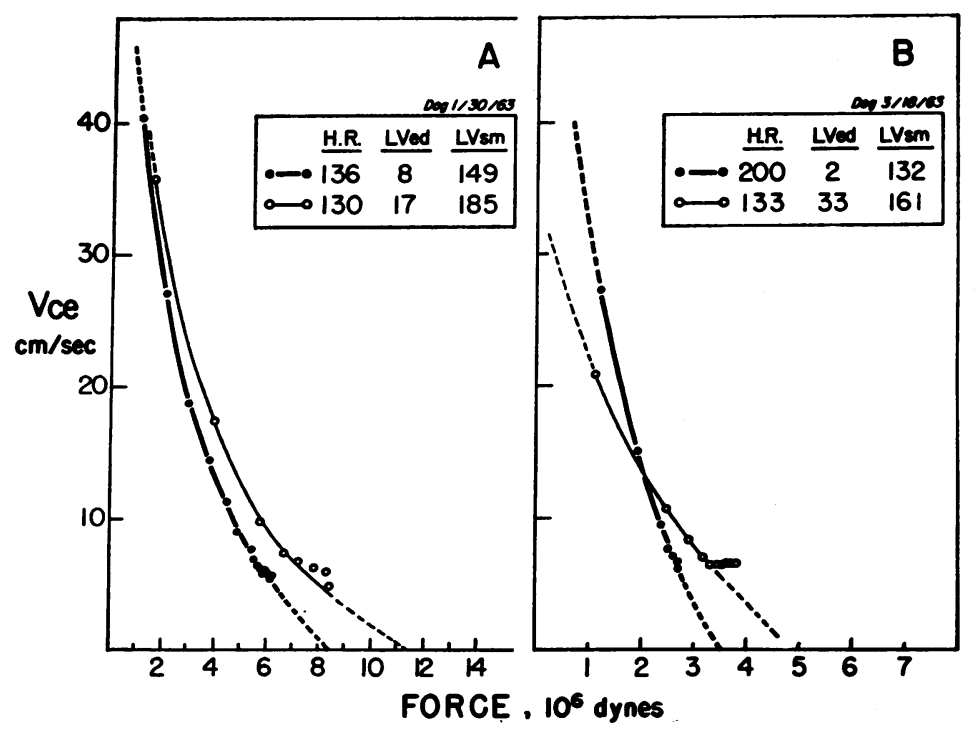

Fig. 9. The EFFECT OF INCREASEd END-DIASTOLIC PRESSURE ON THE F-VCE CURVE. A. LVed pressure is increased without a significant change in heart rate. Note that the projected force at zero velocity $\left(F_{0}\right)$ is increased without an apparent change in the projected velocity at zero load $(V \max )$. B. The elevation in LVed pressure is associated with substantial cardiac slowing. In this instance the increase in projected $F_{0}$ is accompanied by a decrease in the projected $V \max$.

instantaneous points of $\mathrm{F}$ and Vce described an inverse relationship similar to that found in isolated muscle studies $(1,3,4,15,16)$. After peak force was achieved, however, and the contractile effort began to wane, Vce fell as force decreased and the F-Vce relation diverged from the projected inverse curve.

The independent analysis of the force-velocity relation was made in 4 experiments. A minor change in afterload was produced in each by varying the aortic obstruction, and the force-velocity curves derived in the above manner were shown to be virtually unchanged. By an analysis of points of force common to both states, the stiffness of the SEC was calculated and an independent third force-velocity curve constructed that was identical to the other two curves (Figure 8). Because the number of points of force common to both states was small, only a short segment of the independent curve could be constructed. This analysis is, however, not possible when the altered state is associated with a significant change in end-diastolic volume or heart rate.

Effect of increased left ventricular end-diastolic pressure on the F-Vce curve. Six paired experiments were carried out to examine the effect of an increased LVed on the F-Vce curve. In 2 of these the increased LVed was not associated with a significant change in heart rate $(-4.5 \%$ and $-6.5 \%)$, and in both an increase in projected $F_{0}$ was observed without a definite change in projected Vmax (Figure 9-A). In 2 instances the increase in LVed was accompanied by a substantial fall in heart rate $(-30 \%$ and $-33 \%)$, and in both of these an increase in $\mathrm{F}_{\mathrm{o}}$ was associated with a fall in Vmax (Figure 9-B). In the only experiment in this group where heart rate rose $(+14 \%)$ coincident with a rise in LVed, both $\mathrm{F}_{\mathrm{o}}$ and $\mathrm{Vmax}$ increased. In the sixth study severe aortic obstruction was produced; left ventricular systolic mean pressure rose from 139 to $234 \mathrm{~mm} \mathrm{Hg}$ and LVed from 3 to $6 \mathrm{~mm} \mathrm{Hg}$; heart rate remained unchanged. In this instance a rise in both $\mathrm{F}_{\mathrm{o}}$ and $\mathrm{Vmax}$ was observed.

Effects of inotropic intervention on the F-Vce curve. Four paired experiments were performed to examine the effect of isoproterenol on the F-V curve. Because changes in heart rate and LVed were usually produced by isoproterenol, the ef- 


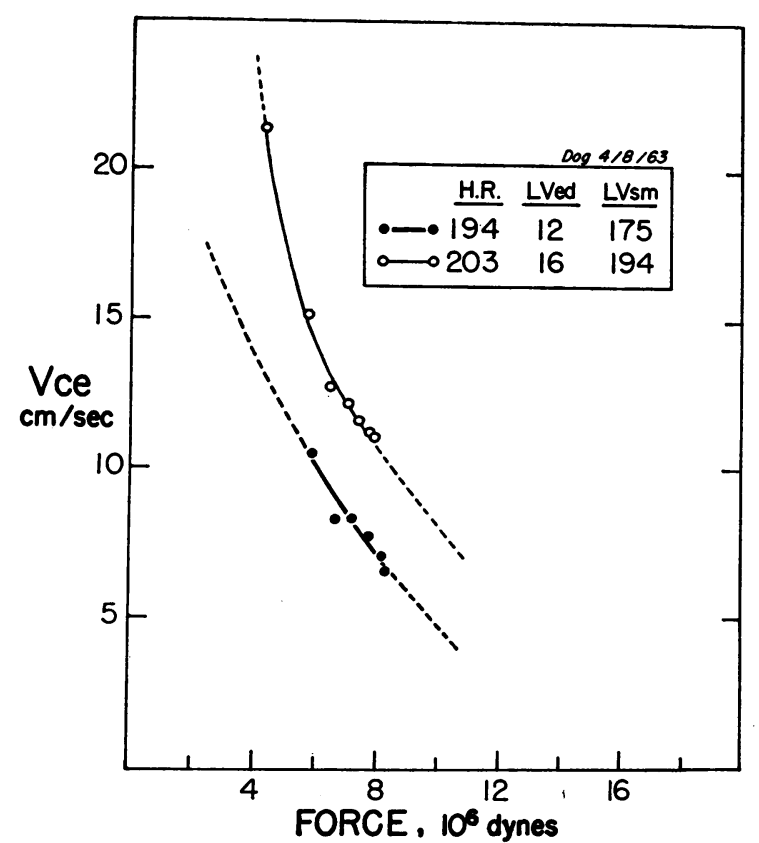

Fig. 10. The efFect of an INOTROPIC Agent on the F-VCE CURVE. Isoproterenol (2.5 $\mu \mathrm{g}$ per minute) produced an increase in the projected values of both $F_{o}$ and Vmax.

fect of inotropism alone was difficult to assess. In one experiment, however, heart rate changed only $5 \%$, and whereas LVed rose slightly from 13 to $15 \mathrm{~mm} \mathrm{Hg}$, the measured end-diastolic volume actually fell by $19 \%$. In this instance the projected values of both $F_{o}$ and $V \max$ were increased by the inotropic agent (Figure 10). Both intercepts of the F-Vce curve were increased in 2 of the remaining 3 experiments, but LVed and end-diastolic volume rose in 1 of these, and in the other heart rate increased $61 \%$. In the fourth experiment, heart rate rose only $10 \%$, and LVed fell slightly from 9 to $7 \mathrm{~mm} \mathrm{Hg}$; only the Vmax was increased.

\section{Discussion}

The contractile effort of muscle is expressed as fiber shortening, force generation, or both. In isotonic contractions of skeletal and cardiac muscle an inverse relation between force and shortening velocity has been demonstrated $(1,3,4,15,16)$. When isometric contractions are analyzed in terms of a series elastic component (Figure 2), an appraisal of force-velocity relations is still possible, but in this case the shortening velocity of the contractile element is equal to the lengthening velocity of the SEC. That there exists in muscle a functional component that behaves like the SEC is supported by the phenomenon of loss of tension during quick release in isometric contractions of skeletal muscle $(17,18)$. The existence of an inverse relation between force and contractile element velocity in isometric contractions can be inferred when the stress-strain relation of the $\operatorname{SEC}(3,14)$ is utilized to derive CE velocity from the rate of force generation.

In our study of intact, beating hearts, the estimation of contractile element velocity is accomplished by summing the instantaneous values of fiber shortening and lengthening velocity of the SEC. Although an inverse relation between $\mathrm{F}$ and Vce was demonstrated in these hearts, an analysis of the important sources of error would seem appropriate.

Sources of error. In these experiments we assumed that the entire stroke volume traversed the aortic obstruction during systole and that the volume of the small reservoir between the aortic valve and the obstruction was constant throughout systole. Gregg has estimated that approximately $25 \%$ of the coronary flow in the dog occurs during systole (19). In our studies the ratio of systolic coronary flow to stroke volume has been estimated to be less than $1: 40$, and thus the error introduced by this factor is probably negligible. Although the constancy of the volume between aortic valve and balloon is not known, an attempt was made to minimize this source of error by proximal positioning of the balloon or coarctation.

Of the measured parameters, the determination of ventricular volume by the thermal dilution technic introduces the largest error, particularly when tis ratio of stroke volume to end-diastolic volume is small. The error is random, however, and a consistent distortion of the derived forcevelocity points is not likely to be introduced by this measurement. This is not necessarily true for the time course of left ventricular volume during systole, and it is not unlikely that flow rate has been underestimated in these studies during early systole due to small variations in effective orifice area and aortic compliance. This underestimation of a change in ventricular volume would overestimate $\mathrm{F}, \mathrm{dF} / \mathrm{dt}$, and $\mathrm{dF} / \mathrm{dl}$ and underestimate 
CFSR during early systole. It is likely, then, that whereas $F$ would be overestimated, Vce would probably appear lower than its true value. However, the resultant error in the slope of the forcevelocity curve depends upon the relative magnitude of these changes, and this is difficult to predict.

The normalized equation used to calculate the stiffness of the SEC in the dog heart was derived from a different species of mammalian heart muscle at a different environmental temperature (14). In spite of this there was reasonably good agreement in the $\mathrm{dF} / \mathrm{dl}$ values at a given force when the independent analysis of $\mathrm{dF} / \mathrm{dl}$ was applied, and Abbott and Mommaerts have found the series elasticity to be independent of temperature change (3).

Other obvious errors are introduced by the complexity of ventricular geometry. All of the calculations utilized in this study assume a spheric, thin-walled, and homogeneous left ventricle. As the fibrous mitral valve leaflets are functionally in series with the ventricular muscle fibers and have unique stress-strain characteristics of their own, the mean stiffness of the ventricular SEC will certainly be altered and thus the derived $\mathrm{dl} / \mathrm{dt}$ and Vce as well. These and other considerations are discussed in greater detail elsewhere (7).

Force-circumferential fiber shortening rate relations. In the absence of outflow obstruction, peak aortic flow is achieved early in systole, and flow is less, for a given left ventricular pressure, during the latter half of systole $(20,21)$. In this manner hysteresis of the pressure flow relationship is produced. When myocardial tensile force is related to CFSR, this hysteresis effect is lessened or even reversed by virtue of a decreasing chamber volume. Thus, in the analysis of the F-CFSR relationship, two opposing effects compete in determining the direction of the loops shown in Figure 3. One is the relative decrease in chamber volume during systole, and the other is the relative time course of left ventricular pressure and flow. Spencer and Greiss have implied that aortic stenosis tends to destroy the hysteresis of the pressure-flow relationship (21). It is not surprising, therefore, that in the F-CFSR relations of the present study, clockwise loops, counterclockwise loops, and loops with no hysteresis were observed. In those instances where clockwise loops were found (CFSR greater for a given $\mathrm{F}$ during early systole), aortic obstruction was usually mild, and the flow curve tended to peak earlier in systole. In the majority of experiments peak flow and CFSR occurred at or even later than peak force, and the F-CFSR loops were counterclockwise.

In terms of muscle mechanics, however, the F-CFSR relation in the intact heart provides useful information only when the SEC is at zero velocity, namely, at peak myocardial tensile force. The interpretation of the mechanics of systole, therefore, rests on the analysis of the forcevelocity curve of the contractile element.

Force-velocity relations. In single, anisotonic, anisometric contractions, an inverse relation between force and contractile element velocity has been demonstrated from the onset of ejection until peak force is achieved. In general when Vce was calculated during the period of isometric contraction, it appeared to approach the projected Vmax along the same force-velocity curve that was constructed from the ejection period of that beat. After the attainment of peak force, the contractile effort appeared to wane, and the forcevelocity relation consistently diverged from the inverse curve. This would suggest that the active state of the muscle (22) is maintained until this point and then is gradually dissipated. ${ }^{8}$ Although the Vce actually becomes negative before the end of the systolic ejection period, this does not imply that the duration of the active state is over, for a decremental restraint on the recoiling SEC is probably still being generated by the CE. Measurements of the force-velocity relationship during the period of isometric relaxation were not made in this study.

There is reason to believe that the forcevelocity curve constructed from a single, normal systole may not be compared to those obtained from isolated, isotonic contractions. In the latter instance velocity is measured at different loads but at the same fiber length and therefore essen-

\footnotetext{
8 In isometric twitches of isolated skeletal muscle, the maximal active state falls off somewhat before peak force is achieved (2). Although we noted variations, we observed no consistent divergence of the F-Vce points from the inverse curve before the point of peak force.
} 


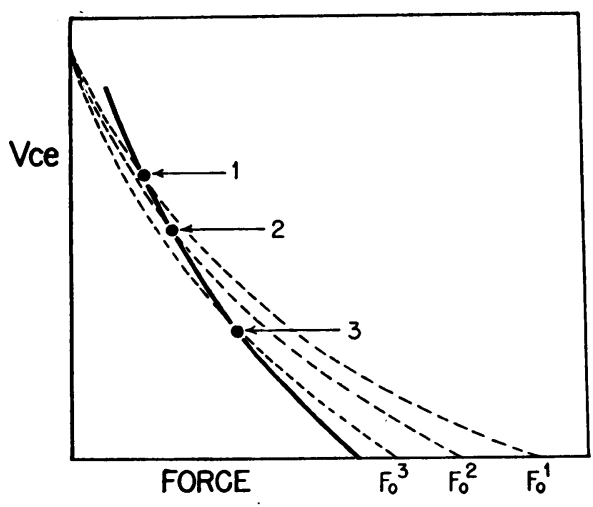

Fig. 11. Family of instantaneous Force-velocity CURVES DURING A SINGLE SYSTOLE (THEORETICAL). The solid curve represents the force-velocity relations of a single systole as derived in the present experiments. As systolic volume becomes progressively smaller during systole, points 1,2 , and 3 are obtained at decreasing $\mathrm{CE}$ lengths. $\mathrm{F}_{0}{ }^{1}, \mathrm{Fo}_{0}{ }^{2}$, and $\mathrm{F}_{0}{ }^{3}$ represent the isometric force that would exist if the $\mathrm{CE}$ length were held constant at each of the three lengths examined. See text.

tially the same CE length. ${ }^{9} \quad$ Abbott and Wilkie have emphasized, however, that when significant shortening of the $\mathrm{CE}$ occurs, the instantaneous relation between $\mathrm{F}$ or Vce will be shifted towards a progressively smaller $F_{0}(23)$. If this is the case, then the force-velocity curve of a single, normal systole represents a composite of forcevelocity relations from a family of curves, each one determined by the instantaneous length of the $\mathrm{CE}$ (Figure 11). This might explain the rather steep curves and high values for Vmax observed in the present study, for at each successive time in systole, the force-velocity point moved to a curve with a smaller $F_{0}$. Indeed, the explanation for the divergence of force-velocity points from the inverse curve during late systole may have more to do with changes in fiber length than with changes in the active state of muscle.

In those experiments in which reasonably good estimates of $\mathrm{F}_{\mathrm{o}}$ could be made, the ratio of $\mathrm{F}$ peak/ $\mathrm{F}_{\mathrm{o}}$ averaged $67 \%$ (50 to $75 \%$ ). Inasmuch as aortic obstruction was present in all of these, the normal, unloaded left ventricle may be expected to function at a lower ratio of $\mathrm{F}$ peak/ $\mathrm{F}_{\mathrm{o}}$. This point is best illustrated by the experiment designed to achieve an independent analysis of

\footnotetext{
${ }^{9}$ Since the change in length of the SEC at different loads will produce a very small absolute change in $\mathrm{CE}$ length, it can probably be ignored.
}

the $\mathrm{dF} / \mathrm{dl}$ values. In this instance a slight increase in afterload was introduced (by varying the aortic obstruction) without producing a change in the force-velocity curve (Figure 8). This was associated with a small increase in contractile element work ${ }^{10}$ at a time when $F$ peak was increased. On theoretical grounds there would appear to be at least two mechanisms by which the heart could increase its contractile element work per beat without a change in the force-velocity curve. The first of these would involve a prolongation of the active state such that the time course of the inverse relation between force and velocity would be extended. It has been suggested that this mechanism may be operative in isolated cardiac muscle contractions (14). An alternative explanation is that the mean product of instantaneous force and velocity (contractile element power) is closer to that portion of the F-Vce curve where maximal power is achieved. In studies of isolated cardiac muscle, work and power have their maxima at about $40 \%$ of $\mathrm{F}_{\mathrm{o}}$ (4). In the present study maximal $\mathrm{CE}$ power was calculated in 12 experiments where $F_{o}$ and $V \max$ could be estimated with some confidence and found to average $33 \%$ (18 to $50 \%$ ). In each of the above two mechanisms, the integrated power curve per beat would yield a larger quantity of work despite the muscle's continuing to function on the same force-velocity curve.

The ability of aortic pressure per se to influence the work of the left ventricle has been noted by a number of investigators (24-28). Sonnenblick and Downing (28) found that the work of the isolated cat papillary muscle at a given muscle length and contractile state was a function of the afterload. Extending these observations to the intact heart, these authors suggested that afterload was "a primary determinant of ventricular performance," independent of inotropic state and initial fiber length. Whether this mechanism plays an important role in determining the performance of the intact heart may depend, at least in part, on which portion of the force-velocity curve the normal heart functions. The full potential of this mechanism may not be realized in the intact heart, however, for a heightened afterload may

10 The calculation of $\mathrm{CE}$ work is presented elsewhere (7). 
increase $F_{0}$ by an increase in end-diastolic fiber length.

The effect of initial fiber length, heart rate, and inotropism on the force-velocity curve. In studies of cat papillary muscle, Sonnenblick found that the effect of an increased preload on the force-velocity curve was such that an increase in $F_{0}$ was produced without a change in $V \max$ (4). The results of our study indicate that the same is true in the intact heart. In each of 6 paired studies, a rise in end-diastolic pressure was associated with an increase in $F_{0}$, and in 5 of the 6 studies Vmax remained unchanged or varied in a manner predictable on the basis of a change in heart rate (vide infra). The analysis of single points on the force-velocity curve (when $\mathrm{dF} /$ $\mathrm{dt}=0$ ) in different dog hearts provides further evidence that $F_{o}$ is increased by an elevated enddiastolic pressure without a change in Vmax (Figure 4). In this analysis the $x$ intercept $\left(\mathrm{F}_{\mathrm{o}}\right)$ of the first order regression line rose in the group with a mild elevation in end-diastolic pressure, whereas the $y$ intercept $(V \max )$ remained unchanged.

Isolated changes in heart rate (without a change in end-diastolic pressure) were rarely observed in the course of this study. Thus, the demonstration of the effect of heart rate on the forcevelocity relationship rests on the analysis of F-CFSR relations at peak force in different dogs and on changes in the force-velocity curve in those studies where both heart rate and end-diastolic pressure were altered. The former analysis merely illustrates that tachysystole is associated with a greater force at a given velocity (Figure 5) but does not indicate whether this came about by virtue of a "force treppe" (increased $F_{0}$ ), a "velocity treppe" (increased Vmax), or both (3, 14). The force-velocity curves constructed from the increased preload experiments, however, are of some help in this regard. When heart rate was unaltered in these experiments, Vmax was not changed. With an increase in heart rate, Vmax rose, and with a decrease in rate, it fell. In only 1 of the 6 studies was an unexplained change in Vmax observed. Thus, a "velocity treppe" was apparently operative at these relatively high heart rates. Since $F_{o}$ was consistently increased by the larger preload, it was impossible to confirm or deny the presence of a "force treppe." In the cat papillary muscle Sonnenblick found a "velocity treppe" over a wide range of altered stimulation frequencies, but the "force treppe" disappeared at higher frequencies (14).

Evaluation of the inotropic effect of isoproterenol too was marred by associated changes in heart rate and end-diastolic pressure. However, in one study in which heart rate did not change and end-diastolic volume actually fell (despite a slight rise in end-diastolic pressure), a rise in both $F_{o}$ and $V \max$ was observed similar to that reported in isolated cardiac muscle (14). In the remaining studies, although an increase in $F_{0}$ or Vmax or both was observed that might reasonably be a manifestation of inotropism, this was not always the sole explanation. Thus, the effect of inotropism on the F-V curve of the intact heart needs further study. To avoid associated changes in heart rate, we have planned a study of the effect of digitalis glycosides.

\section{Summary}

A method is described by which the instantaneous relationship between force and contractile element velocity may be studied in the intact, beating dog heart. Applying the Hill model for muscle contraction, contractile element velocity was derived as the sum of fiber shortening velocity plus the lengthening velocity of the series elastic component. The latter, in turn, was calculated from the time derivative of wall force and the stiffness of the series elastic component. Two methods were employed to estimate the stiffness of the series elastic component of the dog's left ventricle.

From the onset of systolic ejection until peak force is achieved, an inverse relation between force and contractile element velocity was observed, similar to that found in studies of isolated muscle. An increase in end-diastolic pressure was found to alter the force-velocity curve by producing an increase in isometric force without a change in the projected velocity at zero force. The projected velocity at zero force rose and fell with corresponding changes in heart rate, indicating that a velocity treppe was operative at these heart rates. An inotropic agent (isoproterenol) appeared to increase both the isometric force and the projected velocity at zero load. 
Thus, the construction of a force-velocity curve for a single systole provides a means by which cardiac performance may be analyzed in terms of basic muscle mechanics.

\section{Acknowledgments}

The authors wish to acknowledge the technical assistance of Mrs. Gisela Baumann and Miss Alberta Travers and the secretarial aid of Mrs. Judith Ross.

\section{Addendum}

Since this transcript was submitted, Fry, Griggs, and Greenfield have reported a study of force-velocity relations in the beating heart (Myocardial mechanics; tension-velocity-length relationships of heart muscle. Circulat. Res. 1964, 14, 73). In this study, instantaneous tension and muscle segment length were calculated in the minor semiaxis of the left ventricle (assumed to be an ellipsoid), and estimating series elasticity, the investigators derived contractile element velocity. Emphasizing the importance of muscle length upon the force-velocity relations of the heart muscle, they presented evidence of an inverse relation between instantaneous force and velocity at a given left ventricular volume.

\section{References}

1. Hill, A. V. The heat of shortening and the dynamic constants of muscle. Proc. roy. Soc. Lond. B 1938, 126, 136.

2. Hill, A. V. The abrupt transition from rest to activity in muscle. Proc. roy. Soc. Lond. B 1949, 136, 399.

3. Abbott, B. C., and W. F. H. M. Mommaerts. A study of inotropic mechanisms in the papillary muscle preparation. J. gen. Physiol. 1959, 42, 533.

4. Sonnenblick, E. H. Force-velocity relations in mammalian heart muscle. Amer. J. Physiol. 1962, 202, 931.

5. Starling, E. H. The Linacre Lecture on the Law of the Heart. London, Longmans, Green, 1918.

6. Frank, O. Zur Dynamik der Herzmuskels. Z. Biol. 1895, 32, 370.

7. Britman, N. A., and H. J. Levine. Contractile element work: a major determinant of myocardial oxygen consumption. J. clin. Invest. 1964, 43, 1397.

8. Fry, D. L. Discussion of E. H. Sonnenblick. Implications of muscle mechanics in the heart. Fed. Proc. 1962, 21, 991.

9. Peters, J. P., and D. D. Van Slyke. Quantitative Clinical Chemistry. Baltimore, Williams and Wilkins, 1932, vol. 2, p. 321.

10. Rapaport, E., B. D. Wiegand, and J. D. Bristow. Estimation of left ventricular residual volume in the dog by a thermodilution method. Circulat. Res. 1962, 11, 803.

11. Bristow, J. D., R. E. Ferguson, F. Mintz, and E. Rapaport. Thermodilution studies of ventricular volume changes due to isoproterenol and bleeding. J. appl. Physiol. 1963, 18, 129.

12. Daugherty, R. L. Hydraulics. New York, McGrawHill, 1937.

13. Gorlin, R., and S. G. Gorlin. Hydraulic formula for calculation of the area of the stenotic mitral valve, other cardiac valves, and central circulatory shunts. I. Amer. Heart J. 1951, 41, 1.

14. Sonnenblick, E. H. Implications of muscle mechanics in the heart. Fed. Proc. 1962, 21, 975.

15. Fenn, W. O., and B. S. Marsh. Muscular force at different speeds of shortening. J. Physiol. 1935, 85, 277.

16. Ritchie, J. M. The relation between force and velocity of shortening in rat muscle. J. Physiol. (Lond.) 1954, 123, 633.

17. Gasser, H. S., and A. V. Hill. Dynamics of muscular contraction. Proc. roy. Soc. Lond. B 1924, 96, 398.

18. Podolsky, R. J. Kinetics of muscular contraction : the approach to the steady state. Nature (Lond.) 1960, 188, 666.

19. Gregg, D. E. Coronary Circulation in Health and Disease. Philadelphia, Lea \& Febiger, 1950, p. 100.

20. Barnett, G. O., J. C. Greenfield, Jr., and S. M. Fox III. The technique of estimating the instantaneous aortic blood velocity in man from the pressure gradient. Amer. Heart J. 1961, 62, 359.

21. Spencer, M. P., and F. C. Greiss. Dynamics of ventricular ejection. Circulat. Res. 1962, 10, 274.

22. Remington, J. W. Introduction to muscle mechanics, with a glossary of terms. Fed. Proc. 1962, 21, 954.

23. Abbott, B. C., and D. R. Wilkie. The relation between velocity of shortening and the tensionlength curve of skeletal muscle. J. Physiol. (Lond.) 1953, 120, 214.

24. Peserico, E. The influence of mechanical factors of the circulation upon the heart volume. J. Physiol. (Lond.) 1928, 65, 146.

25. Stella, G. The oxygen consumption of the tortoise heart: its dependence upon diastolic volume and on the mechanical conditions of systole. J. Physiol. (Lond.) 1931, 72, 247.

26. Katz, L. N. Analysis of the several factors regulating the performance of the heart. Physiol. Rev. 1955, 35, 91.

27. Sarnoff, S. J., J. H. Mitchell, J. P. Gilmore, and J. P. Remensnyder. Homeometric autoregulation in the heart. Circulat. Res. 1960, 8, 1077.

28. Sonnenblick, E. H., and S. E. Downing. Afterload as a primary determinant of ventricular performance. Amer. J. Physiol. 1963, 204, 604. 